\title{
ON COEFFICIENT MEANS OF CERTAIN SUBCLASSES OF UNIVALENT FUNCTIONS
}

BY

\section{F. HOLLAND AND J. B. TWOMEY}

\begin{abstract}
Let $\mathcal{R}$ denote the class of regular functions whose derivatives have positive real part in the unit disc $\gamma$ and let $\delta$ denote the class of functions starlike in $\gamma$. In this paper we investigate the rates of growth of the means $s_{n}(\lambda)=n^{-1} \sum_{1}^{n}\left|a_{k}\right|^{\lambda}$ $(0<\lambda \leq 1)$ and $t_{n}(\lambda)=n^{-1} \sum_{1}^{n} k^{\lambda}\left|a_{k}\right|^{\lambda}(\lambda>0)$ as $n \rightarrow+\infty$ for bounded $f(z)$ $=\sum_{1}^{\infty} a_{k} z^{k} \in \mathcal{Q P} \cup \delta$. It is proved, for example, that the estimate $t_{n}(\lambda)=\alpha(1)(\log n)^{-\infty(a)}$ $(n \rightarrow+\infty)$, where $\alpha(\lambda)=\lambda / 2$ for $0<\lambda<2$ and $\alpha(\lambda)=1$ for $\lambda \geq 2$, holds for such functions $f$, and that it is best possible for each fixed $\lambda>0$ within the class $\mathcal{Q}$ and for each fixed $\lambda \geq 2$ within the class $\delta$.

It is also shown that the inequality $s_{n}(1)=o(1) n^{-1}(\log n)^{1 / 2}$, which holds for all bounded univalent functions, cannot be improved for bounded $f \in \mathcal{Q}$. The behavior of $t_{n}(\lambda)$ as $n \rightarrow+\infty$ when $a_{k} \geq 0(k \geq 1)$ and $\lambda \geq 1$ is also examined.
\end{abstract}

1. Introduction and statement of results. Let $\mathcal{R}$ denote the class of regular functions of the form

$$
f(z)=\sum_{1}^{\infty} a_{n} z^{n} \quad(|z|<1)
$$

which satisfy $\operatorname{Re} f^{\prime}(z)>0$ in $\gamma=\{z:|z|<1\}$; let $\delta$ denote the class of regular functions (1.1) which are starlike in $\gamma$, and let $\mathcal{L}$ denote the class of functions $f$ for which $f(z)=\log (g(z) / z)(|z|<1)$, where $g \in \delta$. It is well known that if $f \in \mathcal{Q} \cup \delta$, then $f$ is univalent in $\gamma$. It is also well known that $f \in \delta$ iff $h \in \mathcal{Q}$, where $h^{\prime}(z)=z f^{\prime}(z) / f(z)$ and $h(0)=0$, and it follows easily that we also have $f \in \mathcal{L}$ iff $k \in \mathcal{R}$, where

$$
k(z)=\int_{0}^{z}\left[\zeta f^{\prime}(\zeta)+1\right] d \zeta=z+\sum_{n=1}^{\infty} \frac{n}{n+1} a_{n} z^{n+1} .
$$

If $f \in \mathcal{Q}$, then, by a classical result for functions with positive real part, $n\left|a_{n}\right| \leq 2 \operatorname{Re} a_{1}(n \geq 2)$. Applying this result to the function $k$ defined in (1.2), we deduce that if $f \in \mathcal{L}$, then $n\left|a_{n}\right| \leq 2(n \geq 1)$. For $f \in \mathcal{S}$, on the other hand, we have [2] $n\left|a_{n}\right|=O(1)(n \rightarrow+\infty)$ provided $f$ is also bounded in $\gamma$. Combining these results we get

$$
\left|a_{n}\right|=O(1) / n \quad(n \rightarrow+\infty)
$$

Received by the editors March 7, 1972.

AMS (MOS) subject classifications (1970). Primary 30A32, 30A34; Secondary 30A76.

Key words and phrases. Coefficient means, bounded univalent functions, starlike functions, derivatives with positive real part.

Copyright $\odot$ 1974, American Mathematical Society 
for every bounded $f \in \mathcal{Q} \cup \delta \cup \mathcal{L}$. In this paper we investigate the growth of certain means of the coefficients $\left\{a_{n}\right\}$ for bounded $f \in \mathcal{Q} \cup \mathcal{\mathcal { L }}$. We prove the following theorem.

Theorem 1. Let $f(z)=\sum_{1}^{\infty} a_{n} z^{n}$ be regular and bounded in $\gamma$ and suppose that $f \in \mathcal{R} \cup \mathcal{S} \cup \mathcal{L}$. Then, as $N \rightarrow+\infty$,

$$
\begin{aligned}
\sum_{n=1}^{N}\left|a_{n}\right|^{\lambda} & =o(1) N^{1-\lambda}(\log N)^{-\lambda / 2}, & & 0<\lambda<1, \\
& =o(1)(\log N)^{1 / 2}, & & \lambda=1,
\end{aligned}
$$

and

$$
\begin{aligned}
\sum_{n=1}^{N} n^{\lambda}\left|a_{n}\right|^{\lambda} & =o(1) N(\log N)^{-\lambda / 2}, & & 0<\lambda<2, \\
& =o(1) N(\log N)^{-1}, & & \lambda \geq 2 .
\end{aligned}
$$

If, further, $a_{n} \geq 0$ for each $n \geq 1$, then, as $N \rightarrow+\infty$,

$$
\sum_{n=1}^{N} n^{\lambda} a_{n}^{\lambda}=o(1) N(\log N)^{-1}, \quad \lambda \geq 1 .
$$

Inequalities (1.4), (1.5) and (1.6) are best possible within each of the classes CP and $\mathcal{L}$, while (1.6), and hence (1.5) for $\lambda \geq 2$, is best possible within the class $\delta$.

It has already been noted in [4] that inequality (1.4), with $\lambda=1$, is valid for every $f$ in $B$, the class of functions univalent and bounded in $\gamma$, and this of course implies the inequality for bounded $f \in \mathcal{Q} \cup \delta$. Also, the examples discussed in [4] can be used to show that the inequality is best possible within the class $\mathcal{B}$, but this does not imply the stronger result that (1.4) is best possible for bounded $f \in \mathcal{C}$. Whether (1.4) can be improved for the class of bounded starlike functions remains an open question.

In view of (1.6), it seems worth remarking that the functions we construct to show that (1.4) and (1.5) are best possible have real coefficients.

2. Proof of Theorem 1. The positive results. We first prove (1.5). It was shown in [6] that, for bounded $f \in \mathcal{S} \cup \delta$,

$$
\begin{aligned}
2 \pi \sum_{n=1}^{\infty} n^{2}\left|a_{n}\right|^{2} r^{2 n} & =r^{2} \int_{0}^{2 \pi}\left|f^{\prime}\left(r e^{i \theta}\right)\right|^{2} d \theta \\
& =o(1)[(1-r) \log (1 /(1-r))]^{-1},
\end{aligned}
$$

as $r \rightarrow 1$. If $f \in \mathcal{L}$ is bounded in $\gamma$ and $k \in \mathcal{L}$ is defined as in (1.2), then, because of (1.3), $k$ is also bounded in $\gamma$. Applying (2.1) to $k$, we obtain immediately that (2.1) also holds for bounded $f \in \mathcal{L}$. It follows easily from (2.1) that (1.5) holds for $\lambda=2$ and hence also by (1.3) for $\lambda>2$. If $0<\lambda<2$, then by Hölder's inequality 


$$
\begin{aligned}
\sum_{n=1}^{N} n^{\lambda}\left|a_{n}\right|^{\lambda} & \leq\left(\sum_{1}^{N} n^{2}\left|a_{n}\right|^{2}\right)^{\lambda / 2} N^{(2-\lambda) / 2} \\
& =o(1) N(\log N)^{-\lambda / 2},
\end{aligned}
$$

as $N \rightarrow+\infty$, where we have used (1.5) with $\lambda=2$. This completes the proof of (1.5).

We now prove (1.4). Write $T_{n}(\lambda)=\sum_{k=1}^{n} k^{\lambda}\left|a_{k}\right|^{\lambda}$ for $\lambda>0$ and $n \geq 1$. Then by partial summation, for $N \geq 2$,

$$
\begin{aligned}
\sum_{n=1}^{N}\left|a_{n}\right|^{\lambda} & =\sum_{n=1}^{N-1}\left\{n^{-\lambda}-(n+1)^{-\lambda}\right\} T_{n}(\lambda)+N^{-\lambda} T_{N}(\lambda) \\
& \leq \lambda \sum_{n=1}^{N-1} n^{-\lambda-1} T_{n}(\lambda)+N^{-\lambda} T_{N}(\lambda) .
\end{aligned}
$$

By (1.5), we thus have, as $N \rightarrow+\infty$,

$$
\begin{aligned}
\sum_{n=1}^{N}\left|a_{n}\right|^{\lambda} & =o(1) \sum_{n=2}^{N} n^{-\lambda}(\log n)^{-\lambda / 2}+o(1) N^{1-\lambda}(\log N)^{-\lambda / 2} \\
& =o(1) N^{1-\lambda}(\log N)^{-\lambda / 2}
\end{aligned}
$$

for $0<\lambda<1$, and

$$
\begin{aligned}
\sum_{n=1}^{N}\left|a_{n}\right| & =o(1) \sum_{n=2}^{N} n^{-1}(\log n)^{-1 / 2}+o(1)(\log N)^{-1 / 2} \\
& =o(1)(\log N)^{1 / 2}
\end{aligned}
$$

This proves (1.4).

To prove (1.6) we note that it has been shown in [7] that, for bounded $f \in \delta$,

$$
f^{\prime}(r)=\sum_{n=1}^{\infty} n a_{n} r^{n-1}=o(1)\left[(1-r) \log \frac{1}{1-r}\right]^{-1},
$$

as $r \rightarrow 1$, and that it is not too difficult to deduce that (2.3) holds for bounded $f \in \mathcal{P} \cup \mathcal{L}$ as well. If $a_{n} \geq 0$ for $n \geq 1$, then it is an easy consequence of (2.3) that (1.6) holds for $\lambda=1$ and hence also by (1.3) for $\lambda>1$. This proves (1.6).

3. The counter examples. To prove the negative assertions of Theorem 1, we need the following theorem.

Theorem 2. Let $\{\varepsilon(n)\}_{1}^{\infty}$ be a sequence of positive terms which decrease to 0 as $n \uparrow+\infty$. Let $0<\lambda \leq 1$. Then there exist regular functions

$$
f_{\lambda}(z)=1+\sum_{n=1}^{\infty} c_{n}(\lambda) z^{n},
$$

where $c_{n}(\lambda)$ is real for each $n$, for which $\operatorname{Re} f_{\lambda}(z)>0$ in $\gamma$,

$$
\sup _{z \in \gamma}\left|\int_{0}^{z} \frac{f_{\lambda}(\zeta)-1}{\zeta} d \zeta\right|<+\infty,
$$


and, as $N \rightarrow+\infty$,

$$
\begin{aligned}
& S(N, \lambda) \neq O(1) \varepsilon(N) N^{1-\lambda}(\log N)^{-\lambda / 2}, \quad 0<\lambda<1, \\
& S(N, 1) \neq O(1) \varepsilon(N)(\log N)^{1 / 2},
\end{aligned}
$$

where $S(N, \lambda)=\sum_{n=1}^{N} n^{-\lambda}\left|c_{n}(\lambda)\right|^{\lambda}$ for $0<\lambda \leq 1$ and $N \geq 1$.

Proof of Theorem 2. We begin be defining a convex increasing sequence $\left\{n_{k}\right\}_{0}^{\infty}$ of nonnegative even integers for which

$$
k^{5}<n_{k} \leq(k+1)^{5}, \quad k \geq 1
$$

and

(3.4) the equation $n=n_{k} \pm n_{j}(k \geq j \geq 1)$ has at most one solution for each positive integer $n$.

Following Marcinkiewicz [5, p. 43], we define the sequence inductively as follows:

Let $n_{0}=0, n_{1}=2$ and suppose that, for $k \geq 2, n_{0}, n_{1}, \ldots, n_{k-1}$ have been defined. Then there are at most $4 k^{3}$ positive integers of the form $\pm n_{j} \pm n_{l} \pm n_{p}$ where $0 \leq j, l, p \leq k-1$. Hence there is (at least) one even integer, denote it by $n_{k}$, satisfying

$$
(k+1)^{5}-\left(8 k^{3}+2\right) \leq n_{k} \leq(k+1)^{5}
$$

which is not of the form $\pm n_{j} \pm n_{l} \pm n_{p}(0 \leq j, l, p \leq k-1)$. It is easily verified that the increasing sequence $\left\{n_{k}\right\}$ thus defined is convex and satisfies (3.3) and (3.4).

For $|z|<1$ and $m \geq 1$, we now set

$$
F_{m}(z)=\frac{1}{2(m+1)} \sum_{k=0}^{m}\left\{\frac{1+z e^{i \theta_{k}}}{1-z e^{i \theta_{k}}}+\frac{1+z e^{-i \theta_{k}}}{1-z e^{-i \theta_{k}}}\right\}
$$

where $\theta_{0}=0$ and $\theta_{k} \equiv \theta_{k}(m)=\pi n_{k} / 2 n_{m}$ for $1 \leq k \leq m$.

4. To complete the proof of Theorem 2 we need two lemmas.

Lemma 1. Let $F_{m}(z)$ be defined as in (3.5). Then there is an absolute constant $M$ such that

$$
\left|\int_{0}^{z} \frac{F_{m}(\zeta)-1}{\zeta} d \zeta\right| \leq M
$$

for $|z| \leq 1-e^{-m}$ and $m \geq 1$.

Proof. If $G_{m}(z) \equiv \int_{0}^{z}\left(\left(F_{m}(\zeta)-1\right) / \zeta\right) d \zeta$, then, by (3.5),

$$
G_{m}(z)=\frac{1}{m+1} \sum_{k=0}^{m}\left\{l\left(z, \theta_{k}\right)+l\left(z,-\theta_{k}\right)\right\}
$$


where $l(z, \psi)=-\log \left(1-z e^{i \psi}\right)$. Since

$$
|\operatorname{Im} l(z, \psi)| \leq \pi / 2
$$

and

$$
\operatorname{Re} l(z, \psi) \geq \log (1 /(1+r)) \geq-\log 2
$$

for $|z|=r<1$ and $-\pi \leq \psi \leq \pi$, it follows immediately that if $G_{m}(z)=U_{m}(z)$ $+i V_{m}(z)$, then

$$
\left|V_{m}(z)\right| \leq \pi \text { and } U_{m}(z) \geq-2 \log 2
$$

for $|z|<1$ and $m \geq 1$. To complete the proof of (4.1), it is thus sufficient to show that, for $|z| \leq 1-e^{-m}$ and $m \geq 1$,

$$
U_{m}(z)<A,
$$

where $A$ is some absolute constant.

Write $u(r, \psi)=-\log \left|1-r e^{i \psi}\right|$. Then, by (4.2),

$$
U_{m}(z)=\frac{1}{m+1} \sum_{k=0}^{m}\left\{u\left(r, \theta+\theta_{k}\right)+u\left(r, \theta-\theta_{k}\right)\right\},
$$

where $z=r e^{i \theta}$. We prove first that

$$
\sum_{k=0}^{m} u\left(r, \theta-\theta_{k}\right) \leq 2 \sum_{k=0}^{m} u\left(r, \theta_{k}\right)
$$

for $0 \leq r<1$ and $-\pi \leq \theta \leq \pi$. Suppose, to begin with, that $0 \leq \theta \leq \pi / 2$, so that there is an integer $j(1 \leq j \leq m)$ such that $\theta_{j-1} \leq \theta \leq \theta_{j}$. For fixed $r, u(r, \psi)$ is a decreasing function of $\psi$ in $[0, \pi]$, and so

$$
\begin{aligned}
\sum_{k=0}^{m} u\left(r, \theta-\theta_{k}\right) & \leq \sum_{k=0}^{j-1} u\left(r, \theta_{j-1}-\theta_{k}\right)+\sum_{k=j}^{m} u\left(r, \theta_{k}-\theta_{j}\right) \\
& \leq \sum_{k=0}^{j-1} u\left(r, \theta_{j-k-1}\right)+\sum_{k=j}^{m} u\left(r, \theta_{k-j}\right) \\
& \leq 2 \sum_{k=0}^{m} u\left(r, \theta_{k}\right),
\end{aligned}
$$

since the convexity of the sequence $\left\{\theta_{k}\right\}$ implies that $\theta_{n}-\theta_{p} \geq \theta_{n-p}-\theta_{0}=\theta_{n-p}$ for all $p$ and $n$ for which $p \leq n \leq m$. This proves (4.5) for $0 \leq \theta \leq \pi / 2$. It is easy to see that the above argument can be modified to establish (4.5) for the remaining values of $\theta$ in $[-\pi, \pi]$ and we have thus proved (4.5).

It follows from (4.4) and (4.5), and the fact that $u(r, \psi)$ is an even function of $\psi$, that

$$
U_{m}(z) \leq \frac{4}{m+1} \sum_{k=0}^{m} u\left(r, \theta_{k}\right)
$$


for $|z|<1$. But

$$
\left|1-r e^{i \theta_{k}}\right|^{2} \geq 4 r \sin ^{2} \frac{1}{2} \theta_{k} \geq\left(4 r / \pi^{2}\right) \theta_{k}^{2}=r n_{k}^{2} / n_{m}^{2},
$$

and so, if $r=1-e^{-m}$,

$$
\begin{aligned}
\sum_{k=0}^{m} u\left(r, \theta_{k}\right) & \leq u(r, 0)+\sum_{k=1}^{m} \log \frac{n_{m}}{r^{1 / 2} n_{k}} \\
& \leq \log \frac{1}{1-r}+\sum_{k=1}^{m} \log \frac{(m+1)^{5}}{k^{5}}+\frac{m}{2} \log \frac{1}{r} \\
& \leq m+5 m \log (m+1)-5[m \log m-m]+(m / 2) \log 2 \\
& =m\left[6+5 \log ((m+1) / m)+\frac{1}{2} \log 2\right],
\end{aligned}
$$

where we have used (3.3) and the inequality $\sum_{k=1}^{m} \log k \geq m \log m-m$, $(m \geq 1)$. By (4.6) we thus have, for $|z|=1-e^{-m}$,

$$
U_{m}(z) \leq(4 m /(m+1))[6+(11 / 2) \log 2],
$$

and (4.3) follows immediately by the maximum principle for harmonic functions. This proves Lemma 1.

Lemma 2. Let $F_{m}(z)=1+\sum_{1}^{\infty} b_{n}(m) z^{n}$ for $|z|<1$ and $m \geq 1$. Then if $N=2 n_{m}$ and $0<\lambda \leq 2$, we have

$$
\frac{1}{N} \sum_{n=1}^{N}\left|b_{n}(m)\right|^{\lambda} \geq \frac{A(\lambda)}{m^{\lambda / 2}}
$$

where $A(\lambda)$ is a positive constant which depends only on $\lambda$.

Proof (cf. proof of Szidon's Lemma [1, p. 258]). By (3.5) we have

$$
b_{n}(m)=\frac{2}{m+1} \sum_{k=0}^{m} \cos n \theta_{k} .
$$

Hence, writing $b_{n}$ for $b_{n}(m)$ and using the fact that

$$
\cos n \theta_{k} \cos n \theta_{j}=\frac{1}{2}\left\{\cos n\left(\theta_{k}+\theta_{j}\right)+\cos n\left(\theta_{k}-\theta_{j}\right)\right\}
$$

we obtain

$$
\begin{aligned}
(m+1)^{2} b_{n}^{2} & =2 \sum_{j, k=0}^{m}\left\{\cos \frac{n \pi}{N}\left(n_{k}+n_{j}\right)+\cos \frac{n \pi}{N}\left(n_{k}-n_{j}\right)\right\} \\
& =2 \sum_{s=0}^{N} \alpha_{s} \cos \frac{n \pi s}{N},
\end{aligned}
$$

say. We note that $\alpha_{0}=m+2$ and that $\alpha_{s}=0$ when $s$ is odd. It follows easily that 


$$
\frac{1}{N} \sum_{n=1}^{N} b_{n}^{2}=\frac{2^{\alpha_{0}}}{(m+1)^{2}}=\frac{2(m+2)}{(m+1)^{2}} \geq \frac{1}{m},
$$

since

$$
\begin{aligned}
\sum_{n=1}^{N} \cos \frac{\pi x n}{N} & =\operatorname{Re}\left\{\sum_{n=1}^{N} e^{\pi i x n / N}\right\} \\
& =\operatorname{Re}\left\{e^{\pi i x / N} \frac{1-e^{\pi i x}}{1-e^{\pi i x / N}}\right\}=0,
\end{aligned}
$$

when $x$ is an even integer such that $x \neq 0(\bmod 2 N)$.

We note that, by (4.8),

$$
(m+1)^{4} b_{n}^{4}=4 \sum_{s, t=0}^{N} \alpha_{s} \alpha_{t}\left\{\cos \frac{n \pi}{N}(s+t)+\cos \frac{n \pi}{N}(s-t)\right\}
$$

and so, using (4.10) again and the fact that $N$ is even, we obtain

$$
\frac{1}{N} \sum_{n=1}^{N} b_{n}^{4}=\frac{4}{(m+1)^{4}}\left\{\alpha_{0}^{2}+\alpha_{N}^{2}+\sum_{s=0}^{N} \alpha_{s}^{2}\right\}
$$

But, for $s \geq 1$, we have, because of (3.4) and (4.8), $\alpha_{s} \leq 4$ whenever $s=n_{k}+n_{j}$ or $s=\left|n_{k}-n_{j}\right|$ for any $j$ and $k$, and $\alpha_{s}=0$ otherwise. Hence

(4.11) $\frac{1}{N} \sum_{n=1}^{N} b_{n}^{4} \leq \frac{4}{(m+1)^{4}}\left\{2(m+2)^{2}+16+16\left[2(m+1)^{2}\right]\right\} \leq \frac{B}{m^{2}}$,

where $B$ is an absolute constant.

Finally, by Hölder's inequality, for $0<\lambda<2$,

$$
\frac{1}{N} \sum_{n=1}^{N}\left|b_{n}\right|^{2} \leq\left(\frac{1}{N} \sum_{1}^{N}\left|b_{n}\right|\right)^{2 /(4-\lambda)}\left(\frac{1}{N} \sum_{1}^{N}\left|b_{n}\right|^{4}\right)^{(2-\lambda) /(4-\lambda)}
$$

which, by (4.9) and (4.11), implies that

$$
\frac{1}{N} \sum_{n=1}^{N}\left|b_{n}\right|^{\lambda} \geq A(\lambda)\left\{\frac{m^{2(2-\lambda) /(4-\lambda)}}{m}\right\}^{(4-\lambda) / 2}=\frac{A(\lambda)}{m^{\lambda / 2}},
$$

as required. This completes the proof of Lemma 2.

5. We now complete the proof of Theorem 2.

Let $\lambda$ be fixed with $0<\lambda \leq 1$. Choose an increasing sequence $\left\{m_{k}\right\}_{1}^{\infty}$ of positive integers for which

$$
\varepsilon\left(m_{k+1}\right)^{1 / 2 \lambda}<\min \left\{\frac{1}{2} \varepsilon\left(m_{k}\right)^{1 / 2 \lambda}, m_{k}^{-1}\right\}
$$

and

$$
\left(1-r_{k}^{\lambda}\right)^{-1}<m_{k+1},
$$


for $k \geq 1$, where $r_{k}=1-e^{-m_{k}}(k \geq 1)$. We now define

$$
f_{\lambda}(z)=\sum_{k=1}^{\infty} \varepsilon_{k} F_{m_{k}}\left(r_{k} z\right)
$$

for $|z|<1$, where $\varepsilon_{k}=\varepsilon\left(m_{k}\right)^{1 / 2 \lambda}(k \geq 1)$ and $F_{m}(z)$ is defined by (3.5). By (5.1), $\varepsilon_{k+1}<\frac{1}{2} \varepsilon_{k}(k \geq 1)$ and it follows easily that $\sum_{1}^{\infty} \varepsilon_{k}<+\infty$. We assume, as we may, that

$$
\sum_{k=1}^{\infty} \varepsilon_{k}=1
$$

It is clear from (5.3) and (3.5) that $\operatorname{Re} f_{\lambda}(z)>0$ in $\gamma$, and it follows easily from (5.3), (5.4) and (4.1) that (3.1) also holds.

If $f_{\lambda}(z)=1+\sum_{1}^{\infty} c_{n}(\lambda) z^{n}$, then

$$
S(N, \lambda) \equiv \sum_{n=1}^{N} n^{-\lambda}\left|c_{n}(\lambda)\right|^{\lambda}=\sum_{n=1}^{N} n^{-\lambda}\left|\sum_{k=1}^{\infty} \varepsilon_{k} r_{k}^{n} b_{n}\left(m_{k}\right)\right|^{\lambda}
$$

where $\left\{b_{n}(m)\right\}$ is defined as in Lemma 2. Since $|a+b|^{\lambda} \leq 2^{\lambda}\left(|a|^{\lambda}+|b|^{\lambda}\right)$, we have, for $t \geq 2$ and $n \geq 1$,

$$
\begin{aligned}
\left|\sum_{k=1}^{\infty} \varepsilon_{k} r_{k}^{n} b_{n}\left(m_{k}\right)\right|^{\lambda} & \geq 2^{-\lambda}\left[\varepsilon_{t} r_{t}^{n}\left|b_{n}\left(m_{t}\right)\right|\right]^{\lambda}-\left|\left\{\sum_{k=1}^{t-1}+\sum_{k=t+1}^{\infty}\right\} \varepsilon_{k} r_{k}^{n}\right| b_{n}\left(m_{k}\right)||^{\lambda} \\
& \geq 2^{-\lambda}\left[\varepsilon_{t} r_{t}^{n}\left|b_{n}\left(m_{t}\right)\right|\right]^{\lambda}-2^{\lambda}\left[2 r_{t-1}^{n} \sum_{k=1}^{t-1} \varepsilon_{k}\right]^{\lambda}-2^{\lambda}\left[2 \sum_{k=t+1}^{\infty} \varepsilon_{k}\right]^{\lambda}
\end{aligned}
$$

since $\left|b_{n}\left(m_{k}\right)\right| \leq 2$ for $n \geq 1$ and $k \geq 1$. But, for $t \geq 2, \sum_{k=1}^{t-1} \varepsilon_{k} \leq 1$, by (5.6), and, by (5.1),

$$
\sum_{k=t+1}^{\infty} \varepsilon_{k} \leq \varepsilon_{t+1} \sum_{k=1}^{\infty} 2^{-k+1}=2 \varepsilon_{t+1} \leq 2 m_{t}^{-1}
$$

so that

$$
\left|\sum_{k=1}^{\infty} \varepsilon_{k} r_{k}^{n} b_{n}\left(m_{k}\right)\right|^{\lambda} \geq 2^{-\lambda}\left[\varepsilon_{t} r_{t}^{n}\left|b_{n}\left(m_{t}\right)\right|\right]^{\lambda}-4^{\lambda} r_{i-1}^{n \lambda}-8^{\lambda} m_{t}^{-\lambda}
$$

Write now, for $t \geq 1, N_{t}=2 n_{m_{t}}$, where $\left\{n_{k}\right\}$ is the sequence defined in 83 , write $p_{t}=\left[N_{t}^{-1} e^{m_{t}}\right]$ and $P_{t}=p_{t} N_{t}$. Then, by (5.5) and (5.6), for $t \geq 2$,

$$
S\left(P_{t}, \lambda\right) \geq 2^{-\lambda} \varepsilon_{t}^{\lambda} r_{t}^{\lambda P_{t}} \sum_{n=1}^{P_{1}} n^{-\lambda}\left|b_{n}\left(m_{t}\right)\right|^{\lambda}-4^{\lambda} \sum_{n=1}^{\infty} n^{-\lambda} r_{t-1}^{n \lambda}-8^{\lambda} m_{t}^{-\lambda} \sum_{n=1}^{P_{1}} n^{-\lambda}
$$

We note that, as $t \rightarrow+\infty$, we have 


$$
\begin{aligned}
\sum_{n=1}^{\infty} n^{-\lambda} r_{i-1}^{n \lambda} & =O(1)\left(1-r_{t-1}^{\lambda}\right)^{\lambda-1}, & & 0<\lambda<1, \\
& =O(1) \log \left(1-r_{t-1}\right)^{-1}, & & \lambda=1, \\
& =O(1) m_{t}^{1-\lambda}, & & 0<\lambda<1, \\
& =O(1) \log m_{t}, & & \lambda=1,
\end{aligned}
$$

by (5.2), and

$$
\begin{aligned}
\sum_{n=1}^{P_{t}} n^{-\lambda} & =O(1) P_{t}^{1-\lambda}, & & 0<\lambda<1, \\
& =O(1) \log P_{t}, & & \lambda=1 .
\end{aligned}
$$

We note too that, for $0<\lambda \leq 1$ and $t \geq 1$,

$$
\begin{aligned}
r_{t}^{\lambda P_{t}} & \geq \exp \left[-\lambda e^{m_{t}} \log r_{t}^{-1}\right] \\
& =\exp \left[-\lambda \log \left(1-r_{t}\right)^{-1} \log r_{t}^{-1}\right] \geq \exp [-\lambda] .
\end{aligned}
$$

Also, $b_{n}\left(m_{t}\right)$ is a periodic function of $n$ of period $N_{t}$, and so

$$
\begin{aligned}
\sum_{n=1}^{P_{1}} n^{-\lambda}\left|b_{n}\left(m_{t}\right)\right|^{\lambda} & =\sum_{k=0}^{p_{t}-1} \sum_{s=1}^{N_{t}} \frac{\left|b_{s}\left(m_{t}\right)\right|^{\lambda}}{\left[k N_{t}+s\right]^{\lambda}} \\
& \geq \sum_{k=0}^{p_{t}-1}\left[(k+1) N_{t}\right]^{-\lambda} \sum_{s=1}^{N_{t}}\left|b_{s}\left(m_{t}\right)\right|^{\lambda} \\
& \geq A(\lambda) N_{t}^{1-\lambda} m_{t}^{-\lambda / 2} \sum_{k=1}^{p_{t}} k^{-\lambda},
\end{aligned}
$$

by (4.7). Hence, for $t \geq 1$,

$$
\begin{aligned}
\sum_{n=1}^{P_{t}} n^{-\lambda}\left|b_{n}\left(m_{t}\right)\right|^{\lambda} & \geq A_{1}(\lambda) P_{t}^{1-\lambda} m_{t}^{-\lambda / 2} & & 0<\lambda<1, \\
& \geq B_{1} m_{t}^{-1 / 2} \log p_{t}, & & \lambda=1 .
\end{aligned}
$$

Here, and in (5.12) below, $A_{i}(\lambda)(i=1,2,3)$ denote positive constants which depend only on $\lambda$, and $B_{i}(i=1,2,3)$ denote positive absolute constants. By (5.7), (5.8), (5.9), (5.10), (5.11), and the fact that $m_{t}=\log P_{t}+O(1)$ and $\log p_{t}=(1+o(1)) \log P_{t}$ as $t \rightarrow+\infty$, we have

$$
\begin{array}{rrr}
S\left(P_{t}, \lambda\right) \geq A_{2}(\lambda) \varepsilon\left(m_{t}\right)^{1 / 2} P_{t}^{1-\lambda}\left(\log P_{t}\right)^{-\lambda / 2}-A_{3}(\lambda) P_{t}^{1-\lambda}\left(\log P_{t}\right)^{-\lambda}, & 0<\lambda<1, \\
\geq B_{2} \varepsilon\left(m_{t}\right)^{1 / 2}\left(\log P_{t}\right)^{1 / 2}-B_{3} \log \log P_{t}, & \lambda=1,
\end{array}
$$

for $t \geq 2$. Since we may assume without loss of generality that $\{\varepsilon(n)\}$ does not decrease to 0 too rapidly, it is clear that (5.12) implies (3.2). This completes the proof of Theorem 2. 
6. We now show that Theorem 2 implies that (1.4), for $0<\lambda \leq 1$, and (1.5), for $0<\lambda \leq 2$, are both best possible within each of the classes $\mathcal{R}$ and $\mathcal{L}$.

Let $\{\varepsilon(n)\}$ denote a sequence of positive terms which decrease to 0 as $n \uparrow \infty$ and let $0<\lambda \leq 1$. Let $f_{\lambda}$ be defined as in the proof of Theorem 2 and set $g_{\lambda}(z)=\int_{0}^{z} f_{\lambda}(\zeta) d \zeta$ for $z \in \gamma$. Clearly $g_{\lambda} \in \mathcal{C}$. We show next that $g_{\lambda}$ is bounded in $\gamma$. By integration by parts,

$$
g_{\lambda}(z)=z\left\{1+\int_{0}^{2} \frac{f_{\lambda}(\zeta)-1}{\zeta} d \zeta-\int_{0}^{z} \frac{g_{\lambda}(\zeta)-\zeta}{\zeta^{2}} d \zeta\right\}
$$

The first integral on the right of (6.1) is bounded, by (3.1), and the boundedness of the second integral follows easily from the fact that, for $z=r e^{i \theta} \in \gamma$,

$$
\left|g_{\lambda}(z)\right| \leq \int_{0}^{r}\left|f_{\lambda}\left(\rho e^{i \theta}\right)\right| d \rho \leq \int_{0}^{r} \frac{1+\rho}{1-\rho} d \rho<2 \log \frac{1}{1-r}
$$

where we have used a classical inequality for functions with positive real part. Hence $g_{\lambda}$ is bounded in $\gamma$. Now, if $f_{\lambda}(z)=1+\sum_{1}^{\infty} c_{n}(\lambda) z^{n}$ and $g_{\lambda}(z)$ $=\sum_{1}^{\infty} a_{n}(\lambda) z^{n}(z \in \gamma)$, then, for $n \geq 1$,

$$
\left|a_{n+1}(\lambda)\right|=\left|c_{n}(\lambda) /(n+1)\right| \geq \frac{1}{2}\left|c_{n}(\lambda) / n\right| \text {. }
$$

Hence, by (3.2), as $N \rightarrow+\infty$,

$$
\sum_{n=1}^{N}\left|a_{n}(\lambda)\right|^{\lambda} \neq O(1) \varepsilon(N) N^{\lambda-1}(\log N)^{-\lambda / 2}, \text { for } 0<\lambda<1
$$

and

$$
\sum_{n=1}^{N}\left|a_{n}(1)\right| \neq o(1) \varepsilon(N)(\log N)^{1 / 2}
$$

This proves that (1.4) is best possible within the class $\propto R$.

To establish the same result for the class $\mathcal{L}$ we set

$$
h_{\lambda}(z)=\int_{0}^{2} \frac{f_{\lambda}(\zeta)-1}{\zeta} d \zeta \quad(z \in \gamma, 0<\lambda \leq 1),
$$

where $f_{\lambda}$ is defined as above. Then, because of (3.1), $h_{\lambda}$ is bounded in $\gamma$ and, also, it is easily verified that $h_{\lambda} \in \mathcal{L}$. Since $h_{\lambda}(z)=\sum_{1}^{\infty}\left(c_{n}(\lambda) / n\right) z^{n}(z \in \gamma)$, it follows immediately from (3.2) that (1.4) is best possible for the class $\mathcal{L}$ also.

It is an easy consequence of (2.2), and the fact that (1.4) is best possible for $0<\lambda \leq 1$, that (1.5) is also best possible within each of the classes $\mathcal{R}$ and $\mathcal{L}$ for $0<\lambda \leq 1$, and hence for $1<\lambda \leq 2$ as well; since

$$
\frac{1}{N} \sum_{n=1}^{N} n\left|a_{n}\right| \leq\left(\frac{1}{N} \sum_{n=1}^{N} n^{\lambda}\left|a_{n}\right|^{\lambda}\right)^{1 / \lambda}
$$

for $\lambda>1[3$, p. 26]. 
7. It remains to prove that (1.6) is best possible within each of the classes $\subset$, $\delta$ and $\mathcal{L}$ for each fixed $\lambda \geq 1$. To do this we need the following theorem.

Theorem 3. Let $\{\varepsilon(n)\}$ denote a sequence of positive terms which decrease to 0 as $n \uparrow \infty$. Let $\lambda \geq 1$. Then there exists a regular function $g_{\lambda}(z)=1+\sum_{1}^{\infty} b_{n}(\lambda) z^{n}$, where $b_{n}(\lambda)$ is real and nonnegative for each $n$, for which $\operatorname{Re} g_{\lambda}(z)>0(z \in \gamma)$,

$$
\sup _{z \in \gamma}\left|\int_{0}^{2} \frac{g_{\lambda}(\zeta)-1}{\zeta} d \zeta\right|<+\infty
$$

and, as $N \rightarrow+\infty$,

$$
\sum_{n=1}^{N} b_{n}(\lambda)^{\lambda} \neq O(1) \varepsilon(N) N(\log N)^{-1}
$$

Proof. Let $\lambda$ be fixed with $\lambda \geq 1$. Choose an increasing sequence $\left\{n_{k}\right\}_{1}^{\infty}$, of positive integers for which $n_{1}=2$,

$$
\sum_{k=1}^{\infty} \varepsilon\left(n_{k}\right)^{1 / 2 \lambda}<+\infty
$$

and

$$
n_{k} \log n_{k}<\log n_{k+1}, \text { for } k \geq 1 \text {. }
$$

For $n \geq 1$, write $G_{n}(z)=1+2 \sum_{k=1}^{n}(1-k /(n+1)) z^{k}$. It is well known that, for $n \geq 1$, the Fejér kernel $\frac{1}{2} \operatorname{Re} G_{n}\left(e^{i \theta}\right)$ is nonnegative for $\theta \in[-\pi, \pi]$, and it follows immediately that

$$
\operatorname{Re} G_{n}(z)>0 \quad(z \in \gamma, n \geq 1) .
$$

For $z \in \gamma$ we now define

$$
g_{\lambda}(z)=\sum_{k=1}^{\infty} \varepsilon_{k} G_{n_{k}}\left(z^{m_{k}}\right)
$$

where $\varepsilon_{k}=\varepsilon\left(n_{k}\right)^{1 / 2 \lambda}$ and

$$
m_{k}=\log n_{k}
$$

for $k \geq 1$. We assume, as we may, that $\sum_{1}^{\infty} \varepsilon_{k}=1$. Because of (7.4) and (7.7), the power series expansion of $g_{\lambda}(z)$ is obtained by simply removing brackets, that is

$$
\begin{aligned}
g_{\lambda}(z) & =1+\sum_{n=1}^{\infty} b_{n}(\lambda) z^{n} \\
& =1+2 \varepsilon_{1} \sum_{s=1}^{m_{1}}\left(1-\frac{s}{n_{1}+1}\right) z^{s m_{1}}+2 \varepsilon_{2} \sum_{s=1}^{m_{2}}\left(1-\frac{s}{n_{2}+1}\right) z^{s m_{2}}+\cdots
\end{aligned}
$$


Hence, for $z \in \gamma$,

$$
\begin{aligned}
\left|\int_{0}^{2} \frac{g_{\lambda}(\zeta)-1}{\zeta} d \zeta\right| & \leq \sum_{n=1}^{\infty} \frac{b_{n}(\lambda)}{n} \\
& =2 \sum_{k=1}^{\infty} \frac{\varepsilon_{k}}{m_{k}} \sum_{s=1}^{n_{k}}\left(1-\frac{s}{n_{k}+1}\right) / s \\
& <2 \sum_{k=1}^{\infty} \varepsilon_{k} \frac{1+\log n_{k}}{m_{k}}<+\infty
\end{aligned}
$$

by (7.3) and (7.7). This establishes (7.1).

For $k \geq 1$, write $N_{k}=n_{k} m_{k}$. Then, for $k \geq 1$,

$$
\begin{aligned}
\sum_{s=1}^{N_{k}} b_{n}(\lambda)^{\lambda} & \geq 2^{\lambda} \varepsilon_{k}^{\lambda} \sum_{s=1}^{n_{k}}\left(1-\frac{s}{n_{k}+1}\right)^{\lambda} \\
& =\left(\frac{2 \varepsilon_{k}}{n_{k}+1}\right)^{\lambda} \sum_{s=1}^{n_{k}} s^{\lambda} \geq\left(\frac{2 \varepsilon_{k}}{n_{k}+1}\right)^{\lambda} \frac{n_{k}^{\lambda+1}}{\lambda+1} \\
& >\frac{\varepsilon_{k}}{\lambda+1} n_{k}=\frac{\varepsilon\left(n_{k}\right)^{1 / 2 \lambda}}{\lambda+1} N_{k}\left(\log n_{k}\right)^{-1} \\
& >\frac{\varepsilon\left(n_{k}\right)^{1 / 2 \lambda}}{\lambda+1} N_{k}\left(\log N_{k}\right)^{-1},
\end{aligned}
$$

and (7.2) follows immediately. This completes the proof of Theorem 3.

8. It is easily established, by arguments similar to those used in $\S 6$, that Theorem 3 implies that (1.6) is best possible within each of the classes $\mathcal{R}$ and $\mathcal{L}$.

To prove the same result for the class $\delta$ we define, for $z \in \gamma$ and $\lambda \geq 1$,

$$
f_{\lambda}(z)=z \exp \int_{0}^{z} \frac{g_{\lambda}(\zeta)-1}{\zeta} d \zeta
$$

where $g_{\lambda}$ is defined as in the proof of Theorem 3. Then $f_{\lambda} \in \delta, f_{\lambda}$ is bounded in $\gamma$, and $f_{\lambda}(z)=\sum_{1}^{\infty} a_{n}(\lambda) z^{n}(z \in \gamma)$, where $a_{n}(\lambda) \geq 0$ for $n \geq 1$ and $\sum a_{n}(\lambda)$ $<+\infty$. Since $f_{\lambda}(z) / z \neq 0$ for $z$ in the closure of $\gamma$, it follows from Wiener's theorem $\left[1\right.$, p. 194] that the power series expansion of $z / f_{\lambda}(z)$ is also absolutely convergent for $z=1$, i.e.

$$
\frac{z}{f_{\lambda}(z)}=\sum_{0}^{\infty} c_{n}(\lambda) z^{n} \quad(z \in \gamma)
$$

say, where $\sum\left|c_{n}(\lambda)\right|<+\infty$. Now, by (8.1),

$$
g_{\lambda}(z)=\frac{z}{f_{\lambda}(z)} f_{\lambda}^{\prime}(z) \quad(z \in \gamma)
$$

and so, for $n \geq 1$,

$$
b_{n}=\sum_{k=1}^{n+1} c_{n-k+1} k a_{k}
$$


where for brevity we have suppressed $\lambda$. We show now that, for $\lambda \geq 1$ and $N \geq 1$,

$$
\sum_{n=1}^{N} b_{n}^{\lambda} \leq C^{\lambda} \sum_{n=1}^{N+1} n^{\lambda} a_{n}^{\lambda}
$$

where $C=\sum_{0}^{\infty}\left|c_{n}\right|>0$. We note first that, for $\lambda=1,(8.3)$ is an easy consequence of (8.2). Suppose next that $\lambda>1$. Then, by (8.2) and Hölder's inequality,

$$
\begin{aligned}
b_{n}^{\lambda} & \leq \sum_{k=1}^{n+1}\left|c_{n-k+1}\right| k^{\lambda} a_{k}^{\lambda}\left(\sum_{k=0}^{n}\left|c_{k}\right|\right)^{\lambda / \lambda^{\prime}} \\
& \leq C^{\lambda / \lambda^{\lambda}} \sum_{k=1}^{n+1}\left|c_{n-k+1}\right| k^{\lambda} a_{k}^{\lambda}
\end{aligned}
$$

where $\lambda^{\prime}=\lambda /(\lambda-1)$. But

$$
\begin{aligned}
\sum_{n=1}^{N} \sum_{k=1}^{n+1}\left|c_{n-k+1}\right| k^{\lambda} a_{k}^{\lambda} & \leq \sum_{k=0}^{N}\left|c_{k}\right| \sum_{k=1}^{N+1} k^{\lambda} a_{k}^{\lambda} \\
& \leq C \sum_{k=1}^{N+1} k^{\lambda} a_{k}^{\lambda},
\end{aligned}
$$

and this, combined with (8.4), clearly implies (8.3). It is obvious that (8.3) and (7.2) together imply that (1.6), and hence also (1.5) for $\lambda \geq 2$, is best possible within the class $\delta$. We have thus completed the proof of Theorem 1 .

\section{REFERENCES}

1. N. K. Bari, Trigonometric series, Fizmatgiz, Moscow, 1961; English transl., Macmillan, New York; Pergamon Press, Oxford, 1964. MR 23 \# A3411; MR 30 \# 1347.

2. J. Clunie and F. R. Keogh, On starlike and convex schlicht functions, J. London Math. Soc. 35 (1960), 229-233. MR 22 \#1682.

3. G. H. Hardy, J. E. Littlewood and G. Pólya, Inequalities, Cambridge Univ. Press, New York, 1934.

4. P. B. Kennedy and J. B. Twomey, Some properties of bounded univalent functions and related classes of functions, Proc. Roy. Irish Acad. Sect. A 65(1967), 43-49. MR 35 \#4388.

5. J. Marcinkiewicz, Sur la convergence des séries orthogonales, Studia Math. 6(1936), 39-45.

6. J. B. Twomey, On bounded starlike functions, J. Analyse Math. 24 (1971), 191-204.

7. - On the derivative of a starlike function, J. London Math. Soc.(2) 2 (1970), 99-110. MR 40 \#7436.

Department of Mathematics, Universtty College, Cork, Ireland 